Supporting Information for

\title{
Dual-site catalysis of Fe-incorporated oxychlorides as oxygen evolution electrocatalysts
}

Yuto Miyahara, Tomokazu Fukutsuka, Takeshi Abe, and Kohei Miyazaki*

Graduate School of Engineering, Kyoto University

Nishikyo-ku, Kyoto 615-8510, Japan

\section{Contents:}

- Experimental Details

- $\quad$ Supplementary Figure 1 - 14

- Supplementary Table 1 - 8

- References 


\section{Experimental Detail}

\section{Preparation of catalysts}

$\mathrm{LaCoO}_{3}: \mathrm{LaCoO}_{3}$ was prepared by a sol-gel method. ${ }^{\mathrm{S} 1} \mathrm{La}\left(\mathrm{NO}_{3}\right)_{3} \cdot 6 \mathrm{H}_{2} \mathrm{O}(99.9 \%$, Nacalai Tesque), $\mathrm{Co}\left(\mathrm{NO}_{3}\right)_{2} \cdot 6 \mathrm{H}_{2} \mathrm{O}\left(99.5 \%\right.$, Wako Pure Chemical), and $\mathrm{C}_{6} \mathrm{H}_{8} \mathrm{O}_{7}$ (citric acid; 99\%, Nacalai Tesque) (1: 1 : 1 by molar ratio) were dissolved in deionized water $(18.2 \mathrm{M} \Omega)$. The solution was heated at $c a .80^{\circ} \mathrm{C}$ until a viscous gel was obtained. The gel was then calcined at $1023 \mathrm{~K}$ for $5 \mathrm{~h}$ in air and cooled at a rate of $c a .5 \mathrm{~K} \mathrm{~min}^{-1}$.

LaSrCoO 4 : $\mathrm{LaSrCoO}_{4}$ was prepared by a solid-state method. $\mathrm{La}_{2} \mathrm{O}_{3}\left(99.99 \%\right.$, Nacalai Tesque), $\mathrm{SrCO}_{3}$ (99.99\%, Wako Pure Chemical), and $\mathrm{Co}_{3} \mathrm{O}_{4}(99.5 \%$, Aldrich) ( $3: 6: 2$ by molar ratio) were mixed, and heated at $1373 \mathrm{~K}$ for $8 \mathrm{~h}$ in air and cooled at a rate of $c a .5 \mathrm{~K} \mathrm{~min}^{-1}$.

$\mathbf{B a}_{0.5} \mathbf{S r}_{0.5} \mathbf{C o}_{0.8} \mathbf{F e}_{0.2} \mathbf{O}_{3-\delta}$ (BSCF): BSCF was prepared by a sol-gel method. ${ }^{\mathrm{S} 2} \mathrm{Ba}\left(\mathrm{NO}_{3}\right)_{2}(99.9 \%$, Wako Pure Chemical), $\mathrm{Sr}\left(\mathrm{NO}_{3}\right)_{2}$ (98\%, Wako Pure Chemical), $\mathrm{Co}\left(\mathrm{NO}_{3}\right)_{2} \cdot 6 \mathrm{H}_{2} \mathrm{O}$ (99.5\%, Wako Pure Chemical), $\mathrm{Fe}\left(\mathrm{NO}_{3}\right)_{3} \cdot 9 \mathrm{H}_{2} \mathrm{O}\left(99.9 \%\right.$, Wako Pure Chemical), and $\mathrm{C}_{6} \mathrm{H}_{8} \mathrm{O}_{7}$ (citric acid) $(5: 5: 8: 2: 40$ by molar ratio) were dissolved in deionized water (18.2 M $\Omega$ ). Aqueous ammonia (28 wt\%, Nacalai Tesque) was added until the $\mathrm{pH}$ of the solution became $c a$. 7. The solution was heated at $c a .80{ }^{\circ} \mathrm{C}$ until a viscous gel was obtained. The gel was then calcined at $1023 \mathrm{~K}$ for $5 \mathrm{~h}$ in air and cooled at a rate of $c a .5 \mathrm{~K} \mathrm{~min}^{-1}$.

$\mathrm{IrO}_{2}: \mathrm{IrO}_{2}$ was purchased from Sigma-Aldrich (99.9\%), and was used without further treatment.

\section{Measurement of electronic resistivity}

Electronic resistivities of the oxychlorides were measured with a simple four-probe method using Loresta-AX MCP-T370 (Nittoseiko Analytech). Pelletized samples with the size of $\varphi 20 \mathrm{~mm} \times 1 \mathrm{mmt}$ were prepared by uniaxial pressing with the pressure of $20 \mathrm{MPa}$ for $10 \mathrm{~min}$, followed by sintering at $1123 \mathrm{~K}$ for 1 day in air. 


\section{DFT calculations}

The vacancy formation energy was calculated in the standard way,

$$
E_{\mathrm{f}}=E_{\text {def }}+E_{\mathrm{O}_{2}}-E_{\text {perf }}
$$

where $E_{\mathrm{def}}$ is the total energy of a fully relaxed supercell with an oxygen vacancy, $E_{\mathrm{O}_{2}}$ is the total energy of a free oxygen molecule (in a large cell) and $E_{\text {perf }}$ is the total energy of a perfect supercell.

The Gibbs free energy changes for OER steps were calculated using the following equations:

$$
\begin{aligned}
& \Delta G_{1} \\
& =G\left(\mathrm{OH}^{*}\right)-G(*)-\mu_{\mathrm{OH}}=E\left(\mathrm{OH}^{*}\right)-E(*)-E\left(\mathrm{H}_{2} \mathrm{O}\right)+\frac{1}{2} E\left(\mathrm{H}_{2}\right)-e U+\Delta G_{\mathrm{H}}^{+}(\mathrm{pH})+\Delta( \\
& \mathrm{ZPE}-T \Delta S) \\
& \Delta G_{2}=G\left(\mathrm{O}^{*}\right)+\mu_{\mathrm{H}}-G\left(\mathrm{OH}^{*}\right)=E\left(\mathrm{O}^{*}\right)+\frac{1}{2} E\left(\mathrm{H}_{2}\right)-E\left(\mathrm{OH}^{*}\right)-e U+\Delta G_{\mathrm{H}}^{+}(\mathrm{pH})+\Delta(\mathrm{ZPE} \\
& -T \Delta S) \\
& \Delta G_{3}=G\left(\mathrm{OOH}^{*}\right)-G\left(\mathrm{O}^{*}\right)-\mu_{\mathrm{OH}}=E\left(\mathrm{OOH}^{*}\right)-E\left(\mathrm{O}^{*}\right)-E\left(\mathrm{H}_{2} \mathrm{O}\right)-e U+\Delta G_{\mathrm{H}}^{+}(\mathrm{pH})+\Delta(\mathrm{ZPE} \\
& -T \Delta S) \\
& \Delta G_{4}=4 \times\left[1.23 \mathrm{eV}-e U+\Delta G_{\mathrm{H}}^{+}(\mathrm{pH})\right]-\left(\Delta G_{1}+\Delta G_{2}+\Delta G_{3}\right)
\end{aligned}
$$

where $U$ is the electrode potential and the zero point energy (ZPE) and entropy correction values $(T \Delta S)$ are listed in the Supplementary Table 6 . Crystal structure was visualized by VESTA software. ${ }^{\mathrm{S} 3} \mathrm{We}$ chose the (001)- $\mathrm{CoO}_{2}$ termination, which is theoretically considered to be the most active surface for Ruddlesden-Popper catalysts. ${ }^{\mathrm{S}}$ In the present oxychlorides, however, there are still two types of (001)$\mathrm{CoO}_{2}$ termination with either $\mathrm{SrO}$ or $\mathrm{SrCl}$ subsurface. Therefore, we firstly calculated OER free diagrams of $\mathrm{Sr}_{2} \mathrm{CoO}_{3} \mathrm{Cl}$ on the (001)-terminations with both $\mathrm{SrO}$ and $\mathrm{SrCl}$ subsurfaces. Since the resultant theoretical OER overpotentials for $\mathrm{SrO}$ subsurface and $\mathrm{SrCl}$ subsurface were $1.64 \mathrm{~V}$ and 1.80 V (vs. RHE), respectively, we chose the (001)- $\mathrm{CoO}_{2}$ termination with $\mathrm{SrO}$ subsurface, which was more active surface and was in better agreement with the experimental value, for theoretical calculation of $\mathrm{Sr}_{2} \mathrm{Co}_{0.75} \mathrm{Fe}_{0.25} \mathrm{O}_{3} \mathrm{Cl}$. 


\section{Calculation of total electron mole}

Actual surface area of $\mathrm{Sr}_{2} \mathrm{Co}_{0.8} \mathrm{Fe}_{0.2} \mathrm{O}_{3} \mathrm{Cl}$ in catalyst layer is

$$
\begin{gathered}
A_{\text {perovskite }}=\left(250 \mu \mathrm{g} \mathrm{cm}_{\text {disk }}^{-2}\right) \times\left(0.2826 \mathrm{~cm}_{\text {disk }}^{2}\right) \times\left(2.4 \times 10^{-2} \mathrm{~cm}_{\text {perovskite }}^{2} \mu \mathrm{g}^{-1}\right) \\
A_{\text {perovskite }}=1.7 \mathrm{~cm}_{\text {perovskite }}^{2}
\end{gathered}
$$

Total mole of electron flowed during the galvanostatic measurement in Fig. $3 \mathrm{~b}$ can be calculated as follows.

$$
\begin{gathered}
n_{\text {electron }}=\frac{\left(0.1+0.2+0.5+1.0 \mathrm{~mA} \mathrm{~cm}_{\text {perovskite }}^{-2}\right) \times\left(1.7 \mathrm{~cm}_{\text {perovskite }}^{2}\right) \times(900 \mathrm{~s})}{\left(9.65 \times 10^{7} \mathrm{mC} \mathrm{mol}^{-1}\right)} \\
n_{\text {electron }}=2.9 \times 10^{-5} \mathrm{~mol}
\end{gathered}
$$

Mole of $\mathrm{Sr}_{2} \mathrm{Co}_{0.8} \mathrm{Fe}_{0.2} \mathrm{O}_{3} \mathrm{Cl}$ in catalyst layer is

$$
\begin{gathered}
n_{\text {oxychloride }}=\frac{\left(250 \times 10^{-6} \mathrm{~g} \mathrm{~cm}_{\text {disk }}^{-2}\right) \times\left(0.2826 \mathrm{~cm}_{\text {disk }}^{2}\right)}{\left(317.1 \mathrm{~g} \mathrm{~mol}^{-1}\right)} \\
n_{\text {oxychloride }}=2.2 \times 10^{-7} \mathrm{~mol}
\end{gathered}
$$

From the fact that the value of $n_{\text {electron }} / n_{\text {oxychloride }}$ was higher than 100 , we can conclude that the observed current during the galvanostatic measurement was not from an oxidative decomposition of $\mathrm{Sr}_{2} \mathrm{Co}_{0.8} \mathrm{Fe}_{0.2} \mathrm{O}_{3} \mathrm{Cl}$ itself, but from the electrocatalytic oxygen evolution.

\section{Koutecký-Levich plots}

The ORR electron transfer numbers $(n)$ of the composite electrodes were calculated by the KouteckýLevich equation:

$$
\frac{1}{I}=\frac{1}{I_{k}}+\frac{1}{0.62 n F A C_{\mathrm{O}_{2}} D_{\mathrm{O}_{2}}^{2 / 3} v^{-1 / 6} \omega^{1 / 2}}
$$

where $I$ is the measured current, $I_{k}$ is the kinetic current, $n$ is the ORR electron transfer number, $F$ is the Faraday constant $\left(96485 \mathrm{C} \mathrm{mol}^{-1}\right), A$ is the geometric surface area $\left(0.2826 \mathrm{~cm}^{2}\right), C_{\mathrm{O}_{2}}$ is the oxygen concentration in the electrolyte $\left(8.7 \times 10^{-7} \mathrm{~mol} \mathrm{~cm}^{-3}\right), D_{\mathrm{O}_{2}}$ is the oxygen diffusion coefficient $\left(1.53 \times 10^{-5}\right.$ $\left.\mathrm{cm}^{2} \mathrm{~s}^{-1}\right), v$ is the kinematic viscosity $\left(8.7 \times 10^{-3} \mathrm{~cm}^{2} \mathrm{~s}^{-1}\right)$, and $\omega$ is the rotation rate. An ideal $n$ value of 4.0 was obtained for $\operatorname{Pt}(40 \mathrm{wt} \%) /$ Vulcan (E-TEK) from the linear relationship of $I^{-1}$ vs. $\omega^{-1 / 2}$. 


\section{Measurement of diffusion coefficients}

A diffusion coefficient $(D)$ accompanied by redox reactions of Co was determined by Van Buren's electrochemical method. ${ }^{\mathrm{S} 5-\mathrm{S} 7} \mathrm{In}$ the measurement, chronoamperometry was carried out by applying 50 $\mathrm{mV}$ more anodic to the redox potential of Co (an average value of the redox peaks) in a solution of argon-saturated $1.0 \mathrm{~mol} \mathrm{dm}^{-3} \mathrm{KOH}$ solution. Resultant relation between current vs. $t^{-1 / 2}$ was plotted, and linear fitting of the plot was performed to obtain the intercept of the $t^{-1 / 2}$ axis $\left(t_{0}\right)$. An equation $\lambda$ $=a /\left(t_{0} D\right)^{-1 / 2}$, where $\lambda$ is a dimensionless parameter and $a$ is a particle radius, was used for the calculation of $D$. In the present work, $\lambda$ was set at 2 , which represents a rounded parallelepiped (i.e. an intermediate shape between a sphere and a cube). Particle sizes were determined by a relation between surface area $\left(S_{\mathrm{BET}}\right)$ and particle size: $S_{\mathrm{BET}}=6 /(2 a \rho)$, where $\rho$ is the theoretical density of the oxychlorides.

Here, we considered a main diffusion species as proton or hydroxide instead of oxygen ion. This is due to the result that the formation energy of oxygen vacancy was much higher than that of $\mathrm{Ba}_{0.5} \mathrm{Sr}_{0.5} \mathrm{Co}_{0.8} \mathrm{Fe}_{0.2} \mathrm{O}_{3-\delta}$ (Figure $\mathrm{S} 3$ ), suggesting that the resultant high diffusion coefficient could be ascribed to the following reactions with either proton or hydroxide diffusion:

$$
\begin{aligned}
& \mathrm{Sr}_{2} \mathrm{Co}^{3+} \mathrm{O}_{3} \mathrm{Cl}+\mathrm{H}_{2} \mathrm{O}+\mathrm{e}^{-} \rightarrow \mathrm{Sr}_{2} \mathrm{Co}^{2+} \mathrm{O}_{2}(\mathrm{OH}) \mathrm{Cl}+\mathrm{OH}^{-} \\
& \mathrm{Sr}_{2} \mathrm{Co}^{3+} \mathrm{O}_{3} \mathrm{Cl}+\mathrm{OH}^{-} \rightarrow \mathrm{Sr}_{2} \mathrm{Co}^{4+} \mathrm{O}_{2}(\mathrm{OOH}) \mathrm{Cl}+\mathrm{e}^{-}
\end{aligned}
$$


Figures and Tables
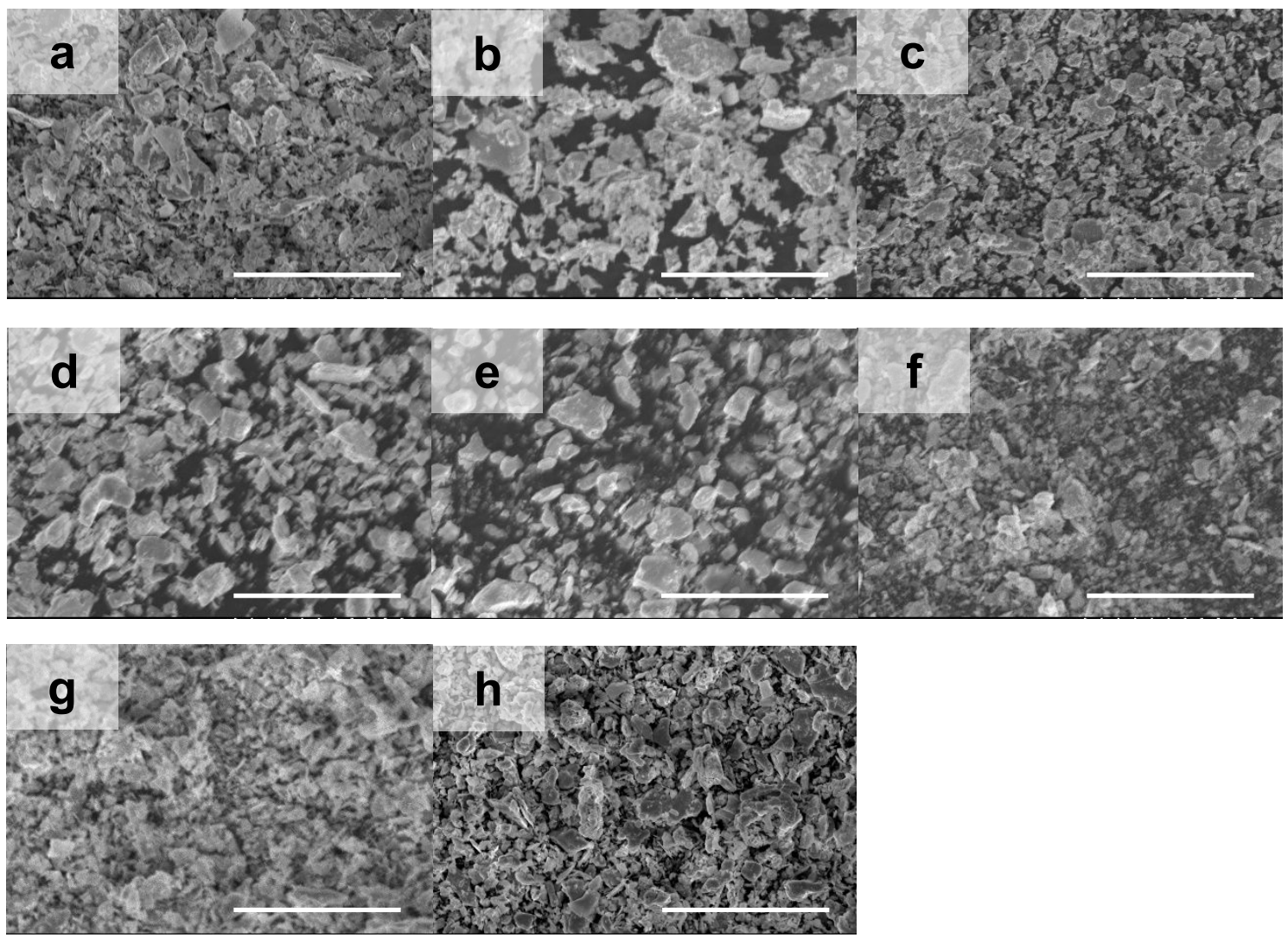

Figure S1. SEM images of $\mathrm{Sr}_{2} \mathrm{Co}_{1-x} \mathrm{Fe}_{x} \mathrm{O}_{3} \mathrm{Cl}$ with (a) $x=0$, (b) 0.1, (c) 0.2, (d) 0.3, (e) 0.4, (f) 0.5, (g) 0.8 and (h) 1.0. White bars represent $50 \mu \mathrm{m}$. 


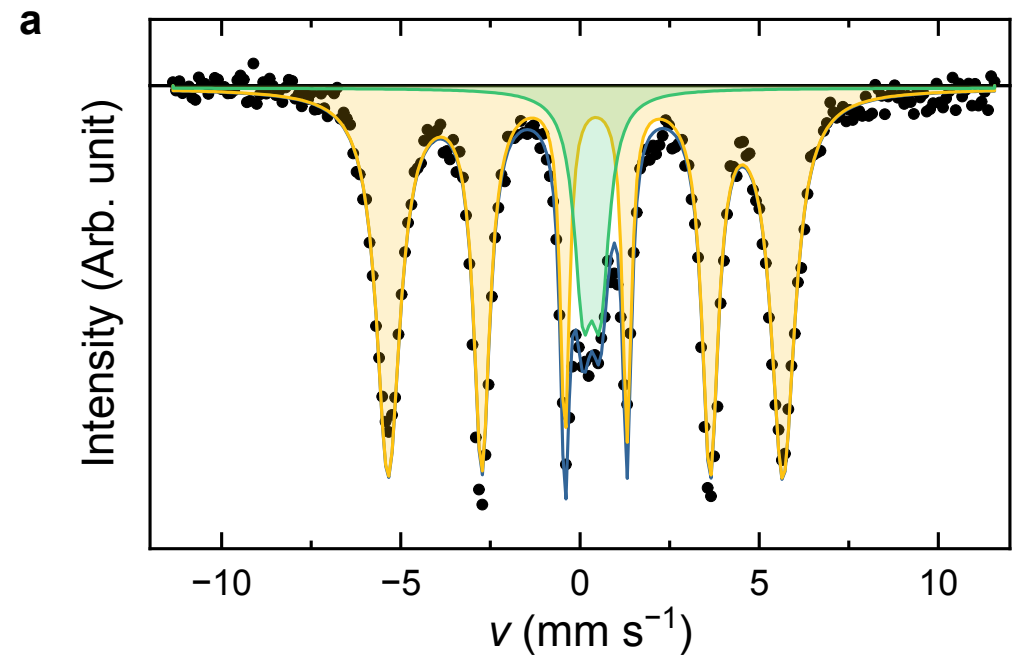

\begin{tabular}{cccccc}
\hline No. & IS / $\mathrm{mm} \mathrm{s}^{-1}$ & $\mathrm{HF} / \mathrm{kOe}$ & $\mathrm{QS} / \mathrm{mm} \mathrm{s}^{-1}$ & Area $/ \%$ & $\mathrm{AS}$ \\
\hline 1 & 0.301 & 341 & -0.290 & 84 & $\mathrm{Fe}^{3+}$ \\
2 & 0.324 & 0 & 0.476 & 16 & $\mathrm{Fe}^{3+}$ \\
\hline
\end{tabular}

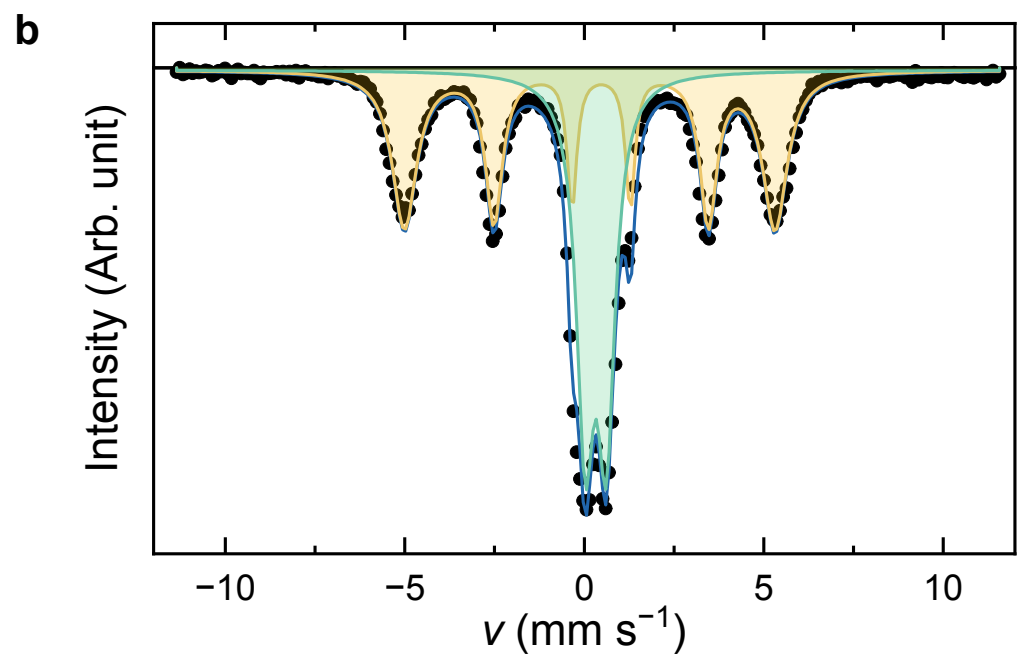

\begin{tabular}{cccccc}
\hline No. & $\mathrm{IS} / \mathrm{mm} \mathrm{s}^{-1}$ & $\mathrm{HF} / \mathrm{kOe}$ & $\mathrm{QS} / \mathrm{mm} \mathrm{s}^{-1}$ & Area $/ \%$ & $\mathrm{AS}$ \\
\hline 1 & 0.311 & 320 & -0.320 & 56 & $\mathrm{Fe}^{3+}$ \\
2 & 0.319 & 0 & 0.476 & 44 & $\mathrm{Fe}^{3+}$ \\
\hline
\end{tabular}

Figure S2. Mössbauer spectra and parameters of (a) $\mathrm{Sr}_{2} \mathrm{Co}_{0.8} \mathrm{Fe}_{0.2} \mathrm{O}_{3} \mathrm{Cl}$ and (b) $\mathrm{Sr}_{2} \mathrm{Co}_{0.5} \mathrm{Fe}_{0.5} \mathrm{O}_{3} \mathrm{Cl}$. IS: isomer shift, HF: magnetic hyperfine field, QS: quadrupole shift, Area: relative area, AS: assignment. 

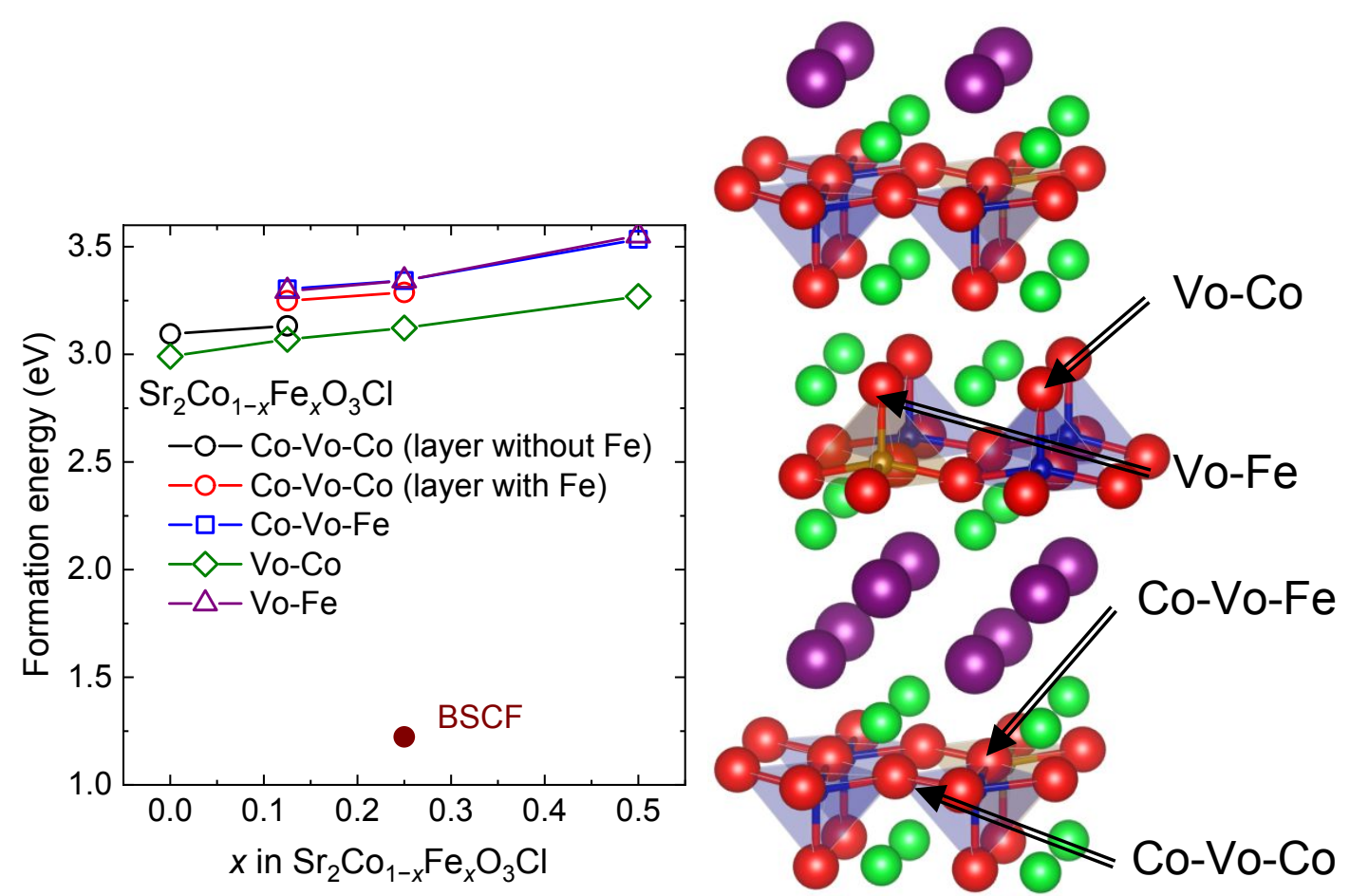

Figure S3. (Left) oxygen vacancy formation energy of $\mathrm{Sr}_{2} \mathrm{Co}_{1-x} \mathrm{Fe}_{x} \mathrm{O}_{3} \mathrm{Cl}$ and (right) schematic illustration of corresponding oxygen vacancy sites. Crystal structure was visualized by VESTA software. ${ }^{3}$ 

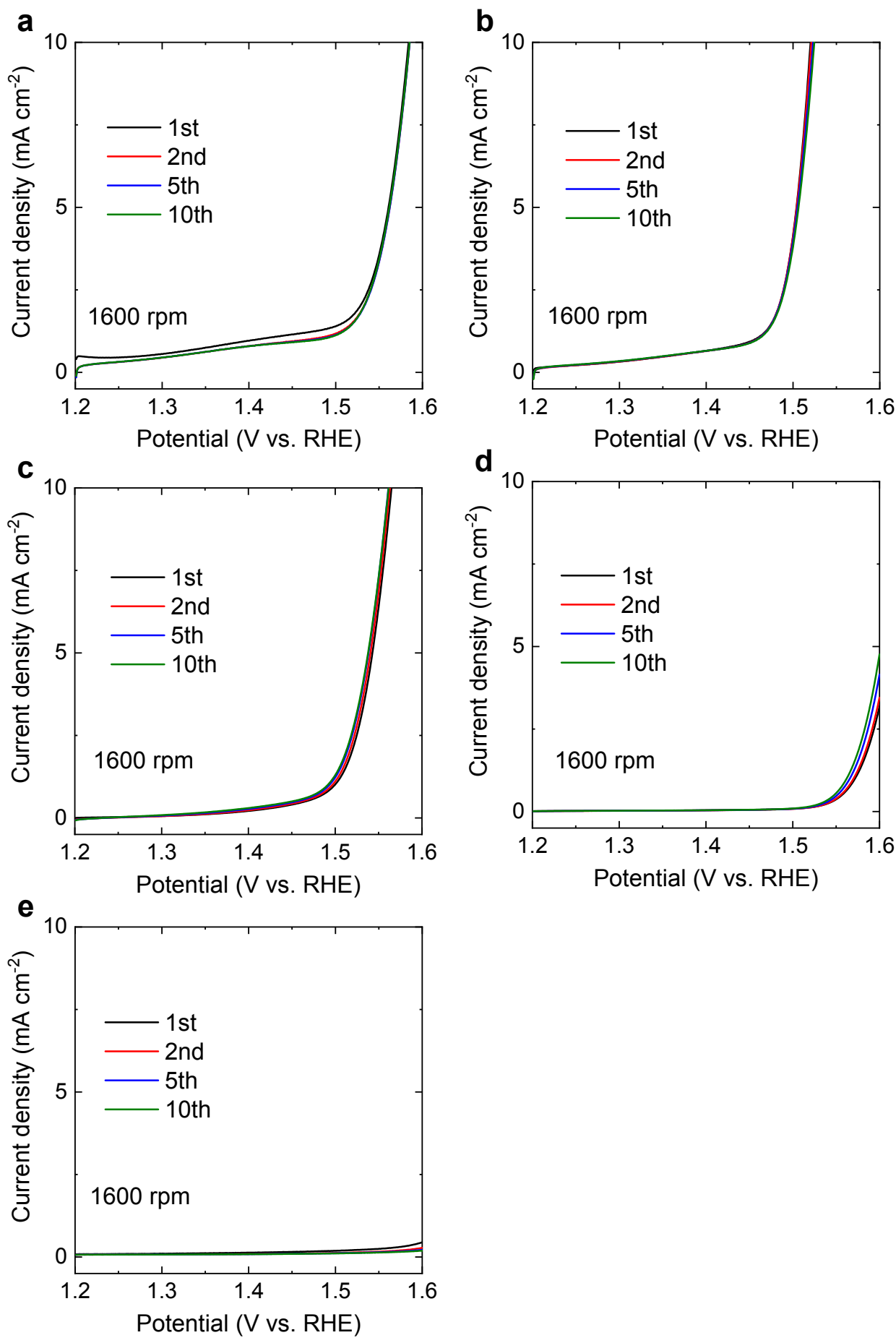

Figure S4. OER polarization curves of $\mathrm{Sr}_{2} \mathrm{Co}_{1-x} \mathrm{Fe}_{x} \mathrm{O}_{3} \mathrm{Cl}$ with (a) $x=0.0$, (b) 0.2, (c) 0.5, (d) 0.8 and (e) 1.0 in an oxygen-saturated $1 \mathrm{M} \mathrm{KOH}$ solution. Rotating speed: $1600 \mathrm{rpm}$. 


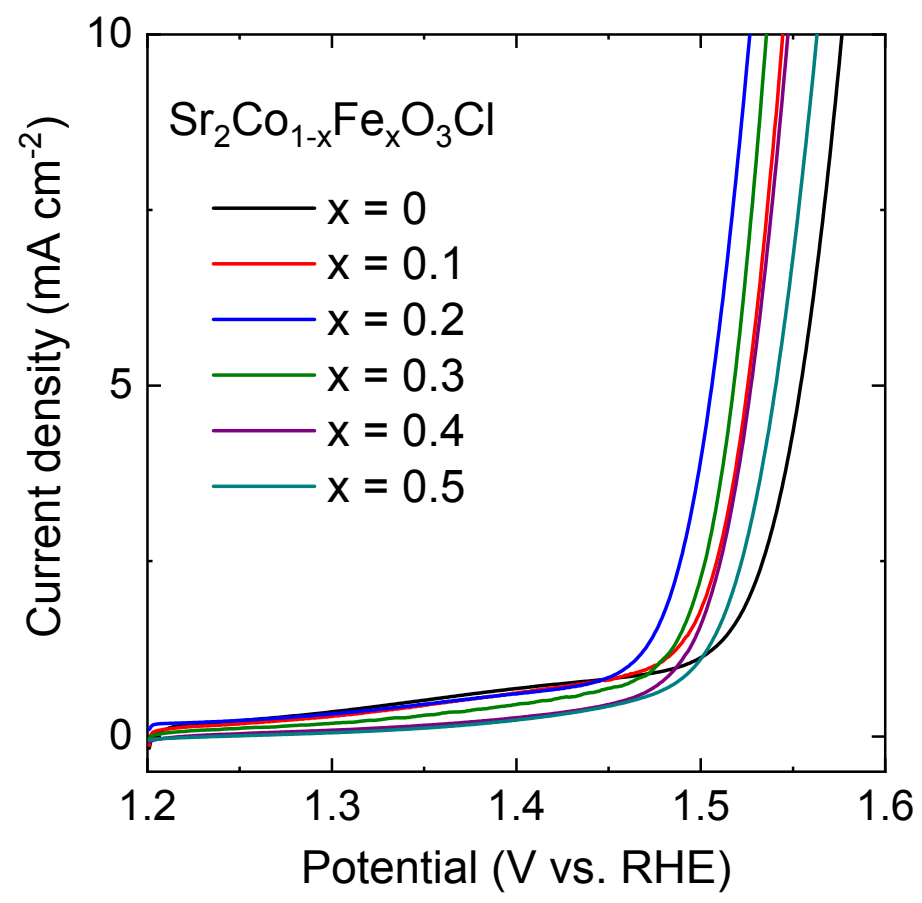

Figure S5. Comparison of OER polarization curves of $\mathrm{Sr}_{2} \mathrm{Co}_{1-x} \mathrm{Fe}_{x} \mathrm{O}_{3} \mathrm{Cl}$ with $x=0 \sim 0.5$ in an oxygensaturated $1 \mathrm{M} \mathrm{KOH}$ solution. Rotating speed: $1600 \mathrm{rpm}$. 

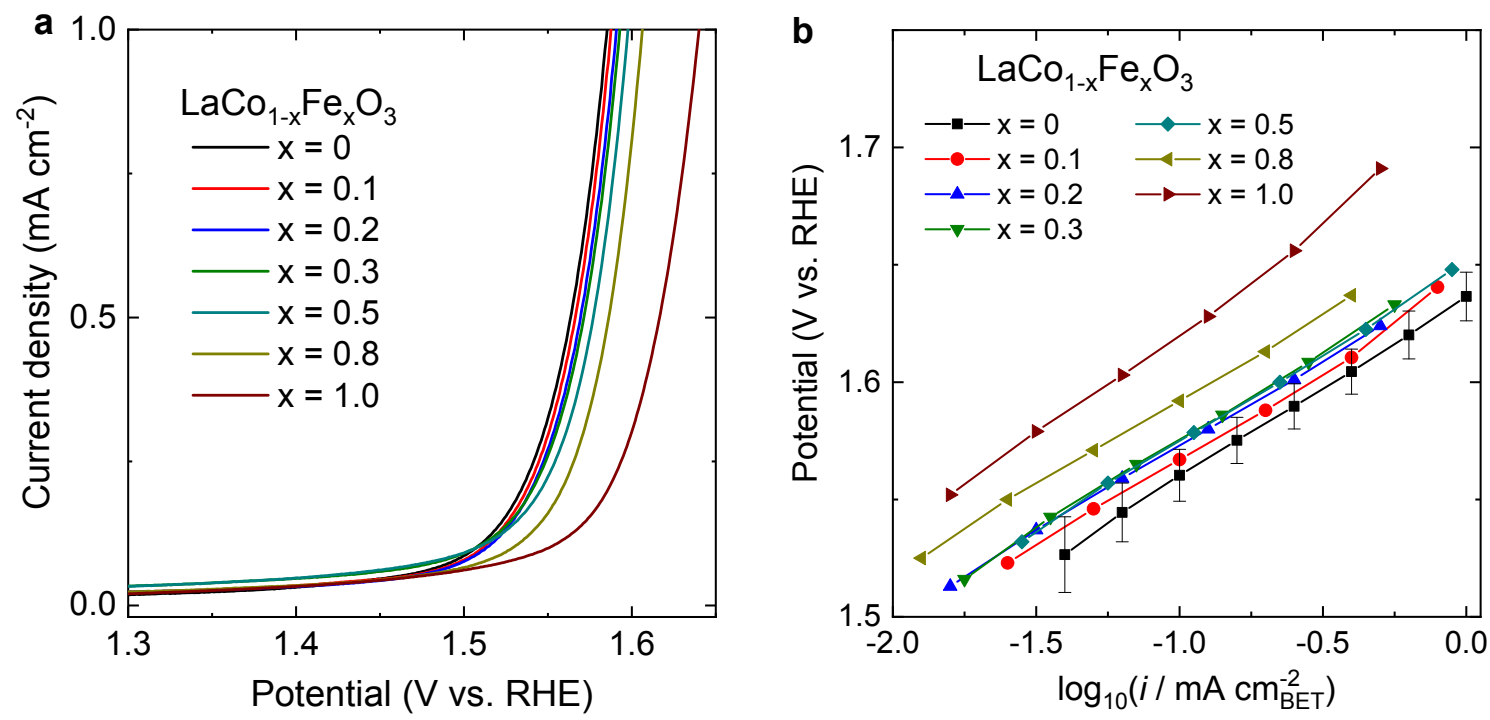

Figure S6. (a) OER polarization curves and (b) corresponding Tafel plots of $\mathrm{LaCo}_{1-x} \mathrm{Fe}_{x} \mathrm{O}_{3}(x=0.0$, $0.1,0.2,0.3,0.5,0.8$ and 1.0) in an oxygen-saturated $1 \mathrm{M} \mathrm{KOH}$ solution. Rotating speed: $1600 \mathrm{rpm}$. 


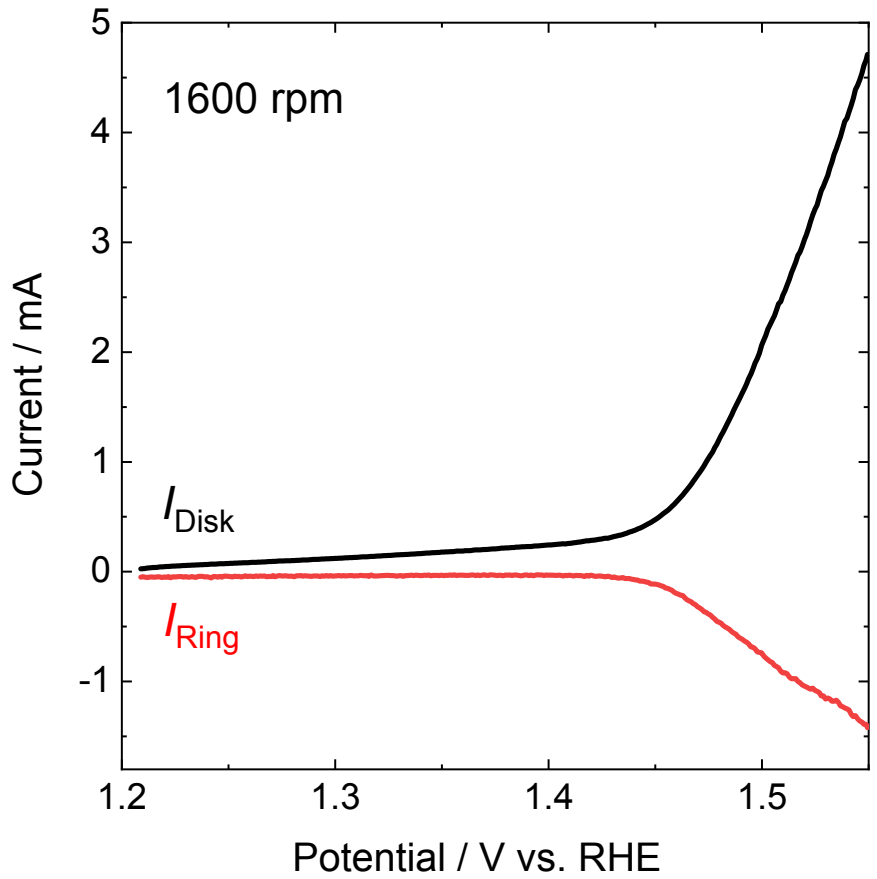

Figure S7. Ring and disk currents of RRDE (Pt ring and GC disk) in an Ar-saturated $1 \mathrm{M} \mathrm{KOH}$ solution at a rotation rate of $1600 \mathrm{rpm}$. Catalyst layer consisting of $\mathrm{Sr}_{2} \mathrm{Co}_{0.8} \mathrm{Fe}_{0.2} \mathrm{O}_{3} \mathrm{Cl}$ : Vulcan : AS-4 $=250: 50: 50 \mu \mathrm{g} \mathrm{cm}^{-2}$ was formed on the GC disk electrode. Scan rate of the disk electrode was set at $10 \mathrm{mV} \mathrm{s}^{-1}$, and the ring electrode was held at $0.4 \mathrm{~V}$ vs. RHE. 


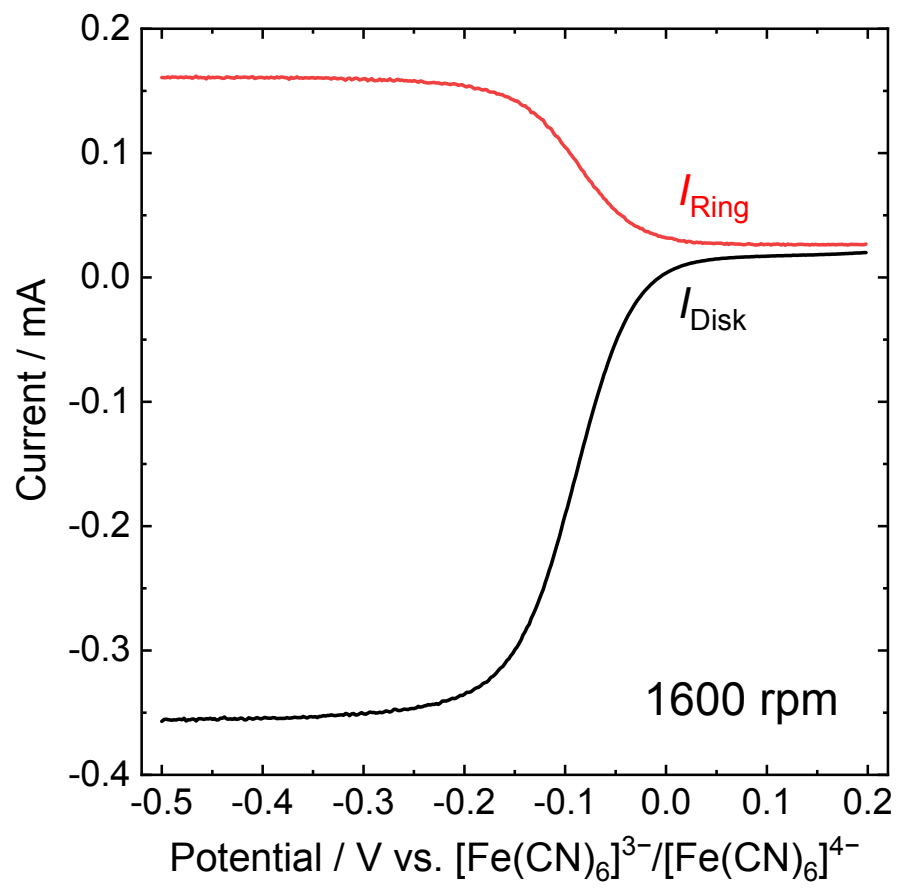

Figure S8. Ring and disk currents of RRDE (Pt ring and GC disk) in an Ar-saturated $2 \mathrm{mM}$ $\mathrm{K}_{3}\left[\mathrm{Fe}(\mathrm{CN})_{6}\right]$ in $1 \mathrm{M} \mathrm{KOH}$ solution at a rotation rate of $1600 \mathrm{rpm}$. Scan rate of the disk electrode was set at $10 \mathrm{mV} \mathrm{s}^{-1}$, and the ring electrode was held at $0.2 \mathrm{~V}$ vs. $\left[\mathrm{Fe}(\mathrm{CN})_{6}\right]^{3-} /\left[\mathrm{Fe}(\mathrm{CN})_{6}\right]^{4-}$. Theoretical collection efficiency was calculated to be $N_{\text {ideal }}=0.374$ (radii of disk, inner ring, and outer ring were $3.0,3.5,4.5 \mathrm{~mm}$, respectively). 


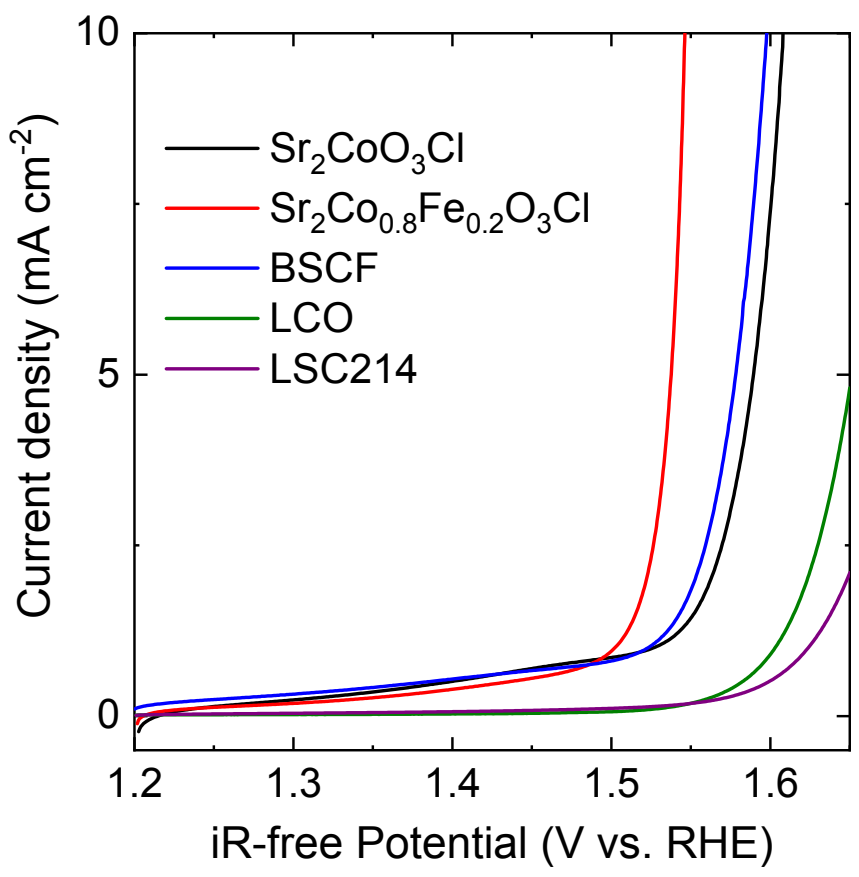

Figure S9. Comparison of OER polarization curves in an oxygen-saturated $0.1 \mathrm{M} \mathrm{KOH}$ solution. Rotating speed: $1600 \mathrm{rpm}$. $i R$ correction $(\sim 28 \Omega)$ were conducted to obtain $i R$-free potentials. 

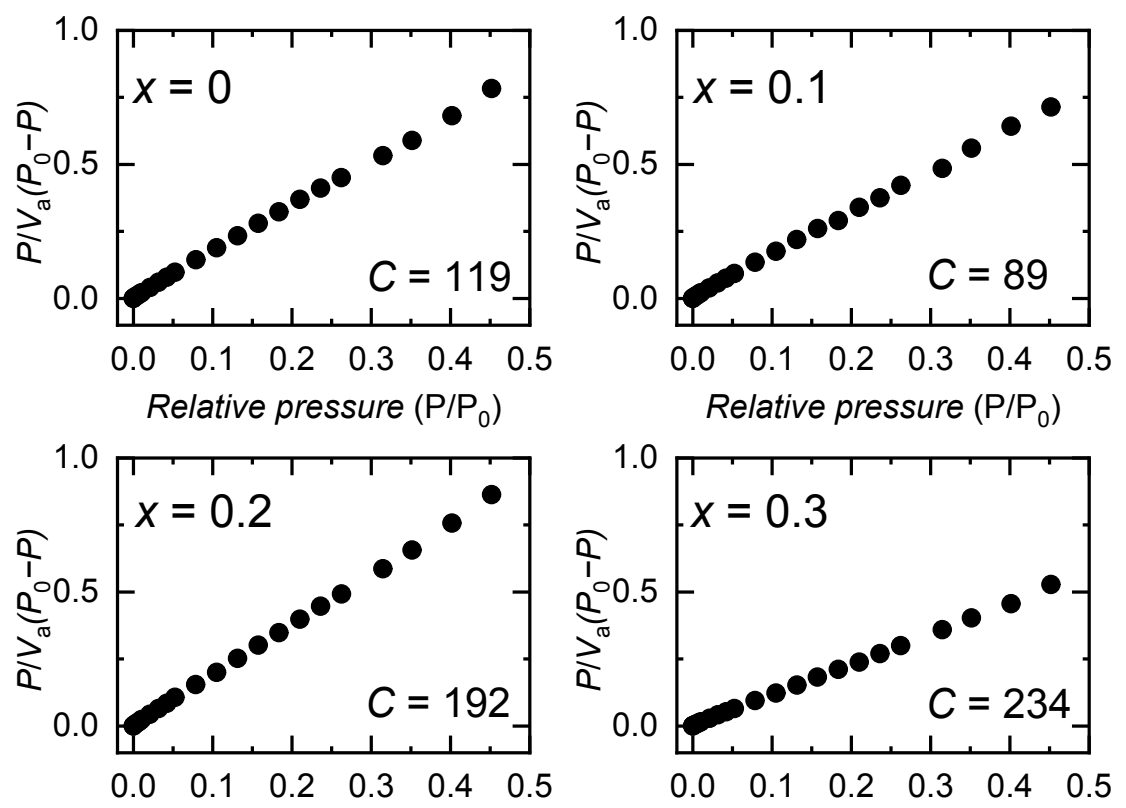

Relative pressure $\left(\mathrm{P} / \mathrm{P}_{0}\right)$

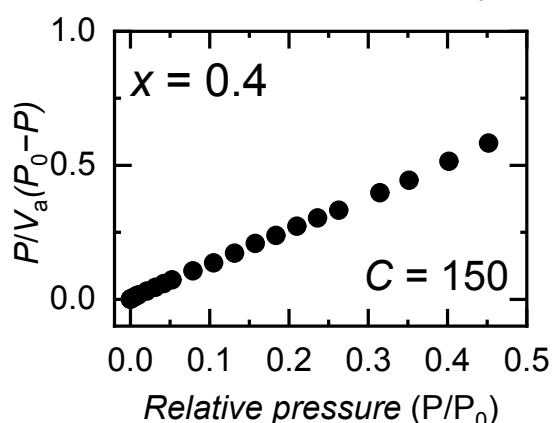

Relative pressure $\left(\mathrm{P} / \mathrm{P}_{0}\right)$
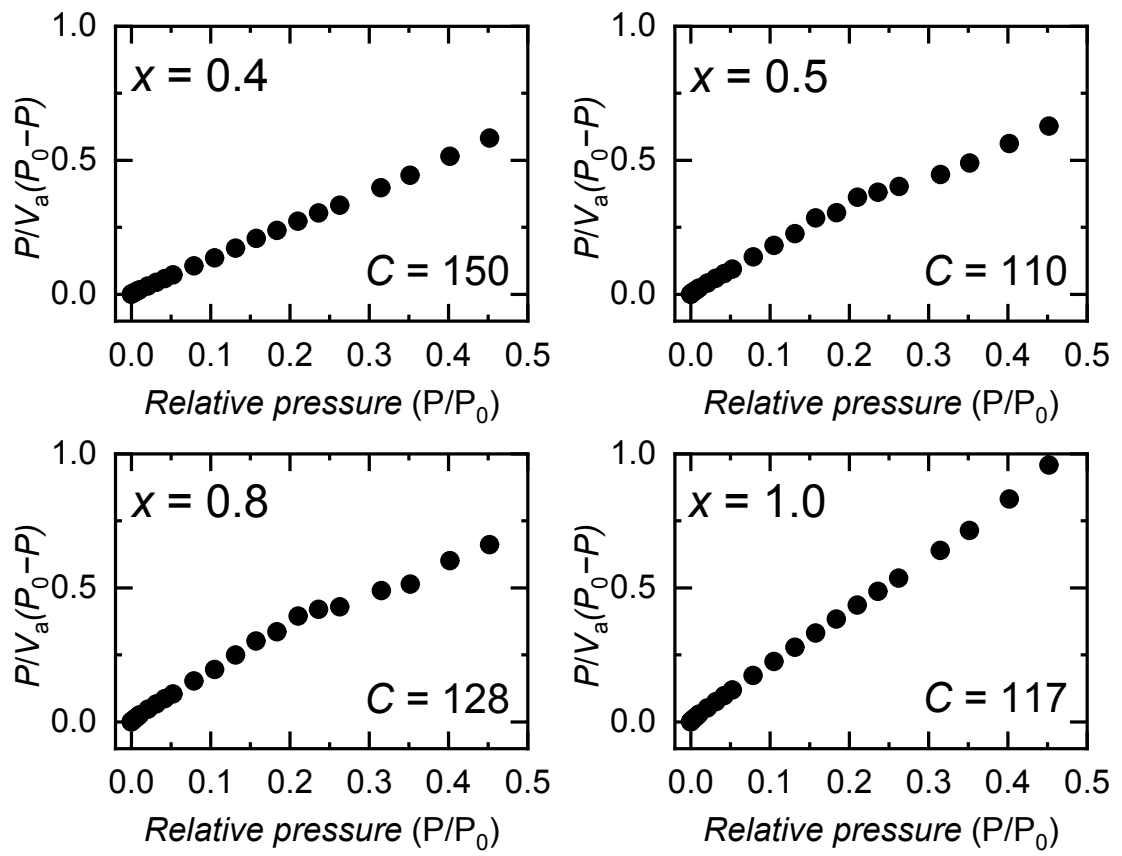

Figure S10. BET plots of $\mathrm{Sr}_{2} \mathrm{Co}_{1-x} \mathrm{Fe}_{x} \mathrm{O}_{3} \mathrm{Cl}$, where $C$ represents their BET constants. 


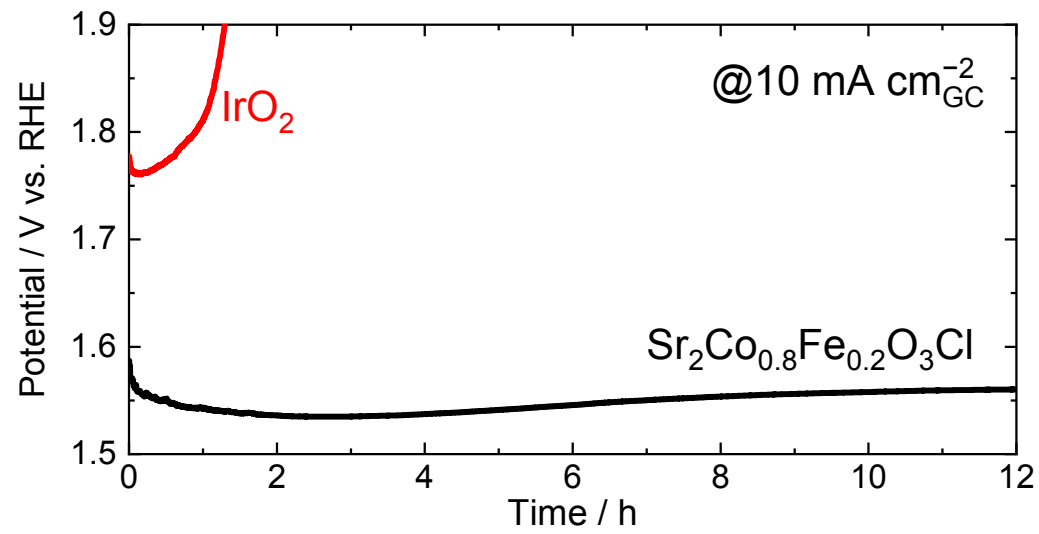

Figure S11. Chronopotentiogram of $\mathrm{Sr}_{2} \mathrm{Co}_{0.8} \mathrm{Fe}_{0.2} \mathrm{O}_{3} \mathrm{Cl}$ at a current density of $10 \mathrm{~mA} \mathrm{~cm}{ }^{-2}$ GC. It should be noted that drastic increase of OER potential for $\mathrm{IrO}_{2}$ from about $1 \mathrm{~h}$ was potentially due to partial cleavage of the catalyst layer. 

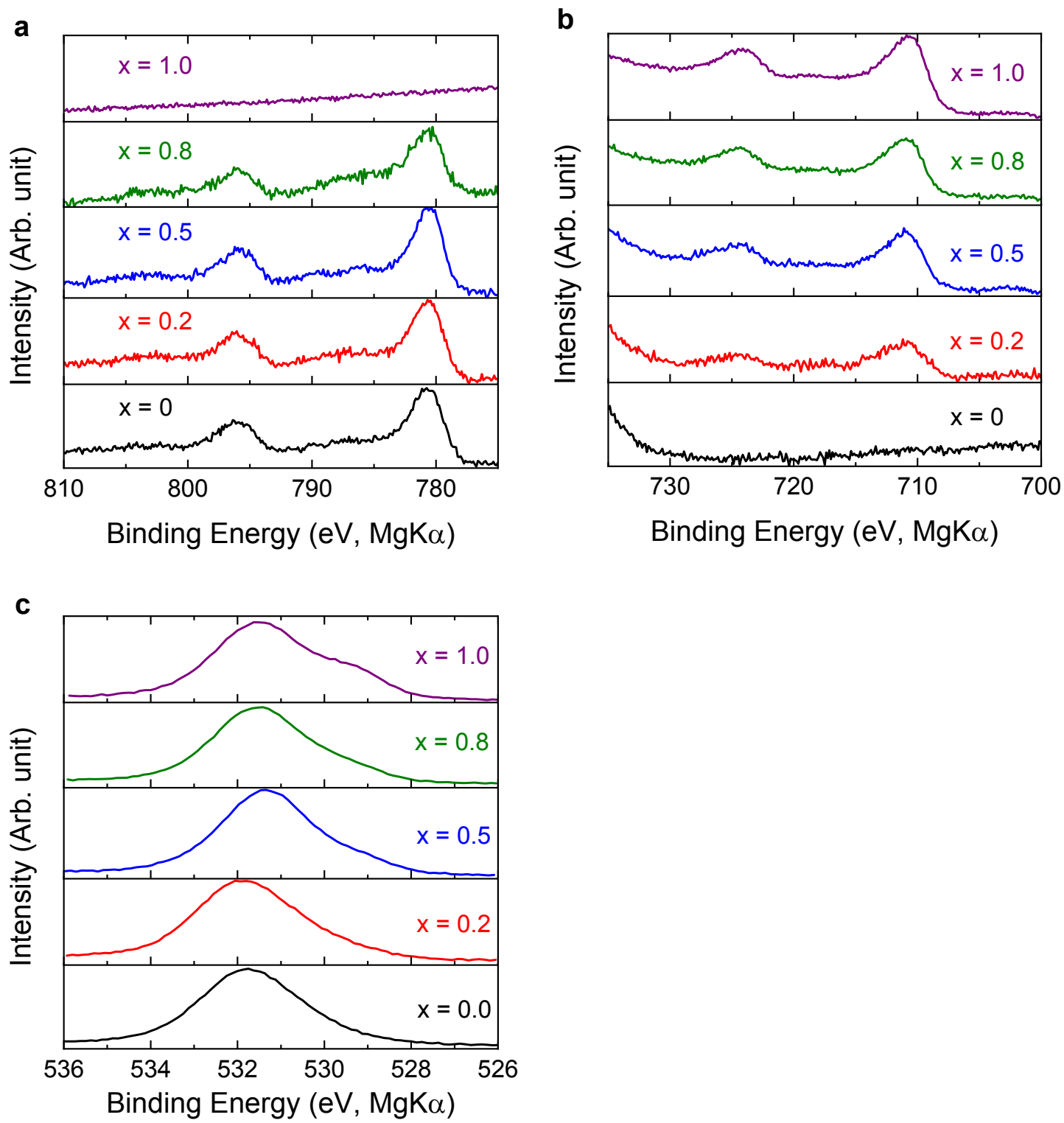

Figure S12. XPS (a) Co $2 p$, (b) Fe $2 p$, and (c) $\mathrm{O} 1 s$ spectra of $\mathrm{Sr}_{2} \mathrm{Co}_{1-x} \mathrm{Fe}_{x} \mathrm{O}_{3} \mathrm{Cl}$. 


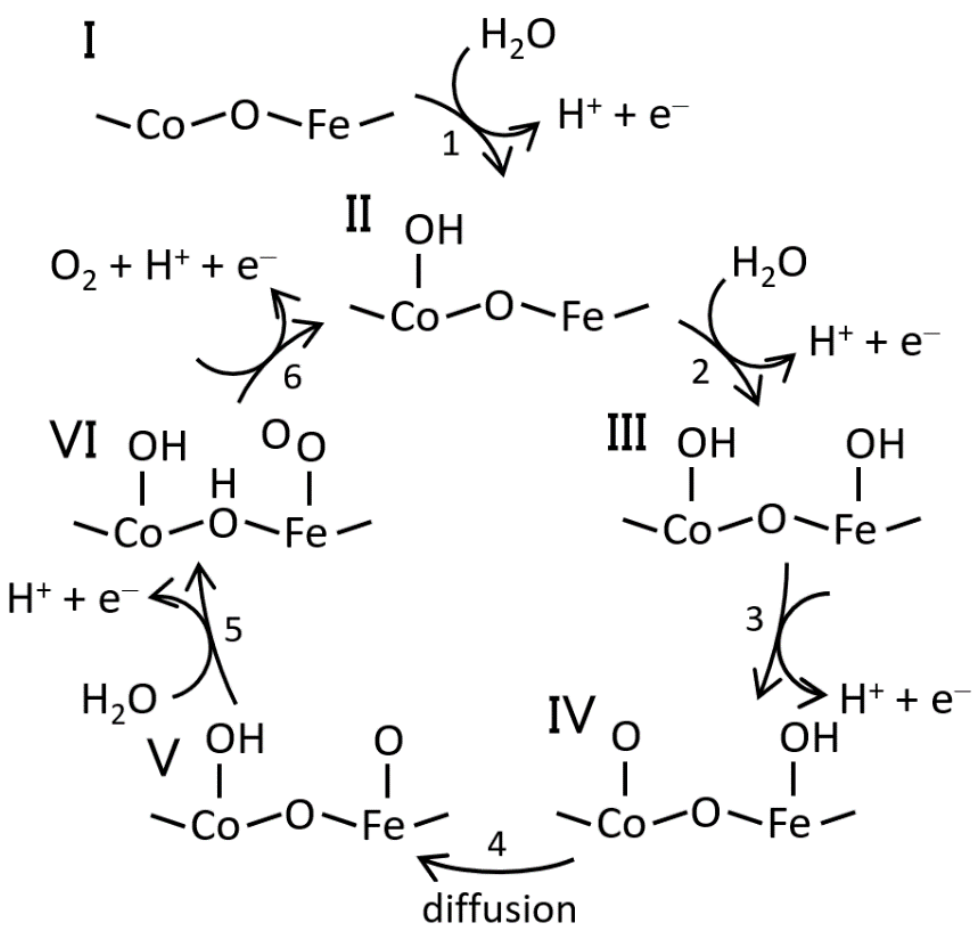

Figure S13. Possible dual-site OER mechanism with hydroxide diffusion from Co to Fe sites in acidic media. It should be noted that the proposed mechanism is conceptual, since $\mathrm{Sr}_{2} \mathrm{Co}_{1-x} \mathrm{Fe}_{x} \mathrm{O}_{3} \mathrm{Cl}$ is found to be chemically unstable in acidic media such as $1 \mathrm{~mol} \mathrm{dm}^{-3} \mathrm{HClO}_{4}$ and $0.5 \mathrm{~mol} \mathrm{dm}^{-3} \mathrm{H}_{2} \mathrm{SO}_{4}$ solutions. 


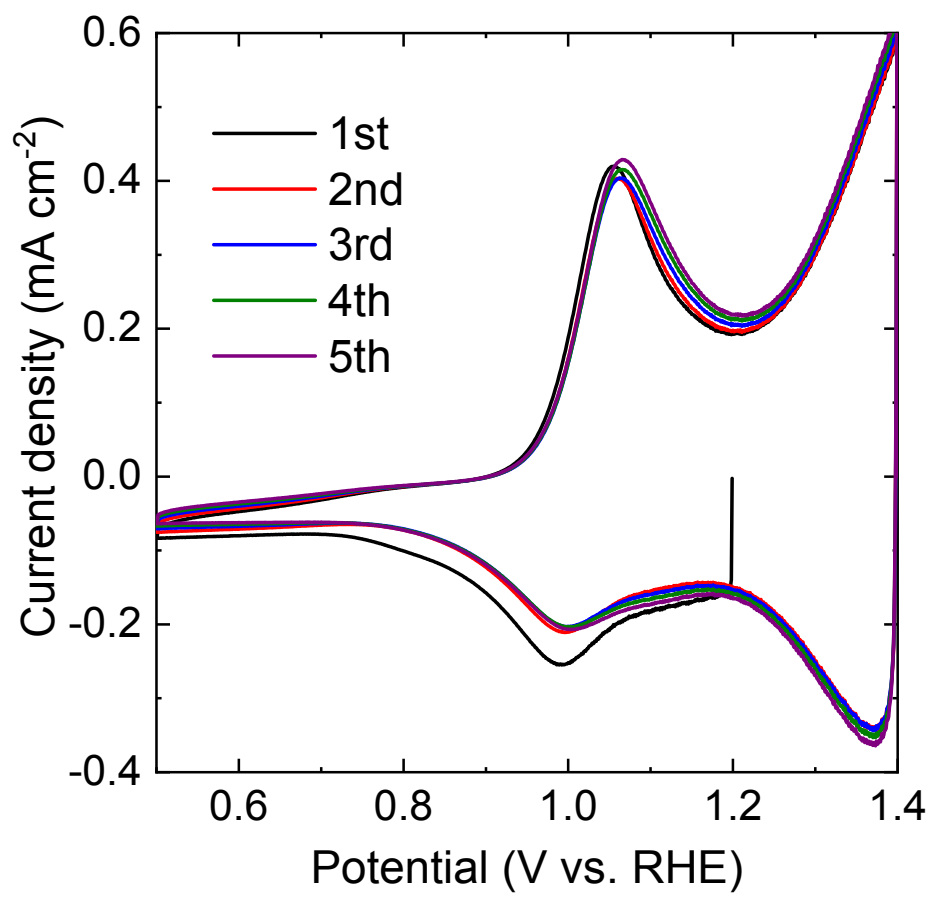

Figure S14. Cyclic voltammogram of $\mathrm{Sr}_{2} \mathrm{CoO}_{3} \mathrm{Cl}$ in an Ar-saturated 1.0 M KOH solution. 
Table S1. Atomic compositions of $\mathrm{Sr}_{2} \mathrm{Co}_{1-x} \mathrm{Fe}_{x} \mathrm{O}_{3} \mathrm{Cl}$ determined by EDX analysis

\begin{tabular}{cc}
\hline Composition & Experimental composition \\
\hline$x=0$ & $\mathrm{Sr}_{1.98} \mathrm{CoO}_{y} \mathrm{Cl}_{0.93}$ \\
$x=0.2$ & $\mathrm{Sr}_{2.07} \mathrm{Co}_{0.78} \mathrm{Fe}_{0.22} \mathrm{O}_{y} \mathrm{Cl}_{1.01}$ \\
$x=0.5$ & $\mathrm{Sr}_{2.02} \mathrm{Co}_{0.50} \mathrm{Fe}_{0.50} \mathrm{O}_{y} \mathrm{Cl}_{0.97}$ \\
$x=0.8$ & $\mathrm{Sr}_{2.03} \mathrm{Co}_{0.21} \mathrm{Fe}_{0.79} \mathrm{O}_{y} \mathrm{Cl}_{0.97}$ \\
$x=1.0$ & $\mathrm{Sr}_{1.98} \mathrm{FeO}_{y} \mathrm{Cl}_{0.95}$
\end{tabular}

Experimental compositions were normalized with nominal $B$ site composition of 1 .

Table S2. Tafel slopes of $\mathrm{Sr}_{2} \mathrm{Co}_{1-x} \mathrm{Fe}_{x} \mathrm{O}_{3} \mathrm{Cl}$, LCO, LSC214, BSCF, and $\mathrm{IrO}_{2}$

\begin{tabular}{cccc}
\hline Composition & Tafel slope & Composition & Tafel slope \\
\hline$x=0.0$ & $60 \mathrm{mV} \mathrm{dec}^{-1}$ & LCO & $74 \mathrm{mV} \mathrm{dec}^{-1}$ \\
$x=0.2$ & $50 \mathrm{mV} \mathrm{dec}^{-1}$ & LSC214 & $88 \mathrm{mV} \mathrm{dec}^{-1}$ \\
$x=0.5$ & $55 \mathrm{mV} \mathrm{dec}^{-1}$ & $\mathrm{BSCF}$ & $72 \mathrm{mV} \mathrm{dec}^{-1}$ \\
$x=0.8$ & $60 \mathrm{mV} \mathrm{dec}^{-1}$ & $\mathrm{IrO}_{2}$ & $86 \mathrm{mV} \mathrm{dec}^{-1}$ \\
$x=1.0$ & $80 \mathrm{mV} \mathrm{dec}^{-1}$ & & \\
\hline
\end{tabular}


Table S3. BET surface areas of $\mathrm{Sr}_{2} \mathrm{Co}_{1-x} \mathrm{Fe}_{x} \mathrm{O}_{3} \mathrm{Cl}$ and benchmarking catalysts

\begin{tabular}{cc}
\hline Sample & Surface area $\left(\mathrm{m}^{2} \mathrm{~g}^{-1}\right)$ \\
\hline $\mathrm{Sr}_{2} \mathrm{CoO}_{3} \mathrm{Cl}$ & 2.6 \\
$\mathrm{Sr}_{2} \mathrm{Co}_{0.9} \mathrm{Fe}_{0.1} \mathrm{O}_{3} \mathrm{Cl}$ & 2.8 \\
$\mathrm{Sr}_{2} \mathrm{Co}_{0.8} \mathrm{Fe}_{0.2} \mathrm{O}_{3} \mathrm{Cl}$ & 2.4 \\
$\mathrm{Sr}_{2} \mathrm{Co}_{0.7} \mathrm{Fe}_{0.3} \mathrm{O}_{3} \mathrm{Cl}$ & 3.8 \\
$\mathrm{Sr}_{2} \mathrm{Co}_{0.6} \mathrm{Fe}_{0.4} \mathrm{O}_{3} \mathrm{Cl}$ & 3.5 \\
$\mathrm{Sr}_{2} \mathrm{Co}_{0.5} \mathrm{Fe}_{0.5} \mathrm{O}_{3} \mathrm{Cl}$ & 2.7 \\
$\mathrm{Sr}_{2} \mathrm{Co}_{0.2} \mathrm{Fe}_{0.8} \mathrm{O}_{3} \mathrm{Cl}$ & 2.5 \\
$\mathrm{Sr}_{2} \mathrm{FeO} \mathrm{O}_{3} \mathrm{Cl}$ & 2.2 \\
$\mathrm{LSC}_{214}$ & 1.4 \\
$\mathrm{LCO}$ & 2.0 \\
$\mathrm{BSCF}$ & 1.5 \\
$\mathrm{IrO}$ & 0.8 \\
\hline
\end{tabular}

Table S4. ORR, OER, and sum overpotentials of perovskites

\begin{tabular}{cccc}
\hline & $\eta_{\mathrm{ORR}}\left(-50 \mu \mathrm{A} \mathrm{cm}_{\mathrm{BET}^{-2}}\right)$ & $\eta_{\mathrm{OER}}\left(100 \mu \mathrm{A} \mathrm{cm}_{\mathrm{BET}^{-2}}\right)$ & $\eta_{\mathrm{SUM}}$ \\
\hline $\mathrm{Sr}_{2} \mathrm{CoO}_{3} \mathrm{Cl}$ & $0.46 \mathrm{~V}$ & $0.28 \mathrm{~V}$ & $0.74 \mathrm{~V}$ \\
$\mathrm{Sr}_{2} \mathrm{Co}_{0.8} \mathrm{Fe}_{0.2} \mathrm{O}_{3} \mathrm{Cl}$ & $0.46 \mathrm{~V}$ & $0.23 \mathrm{~V}$ & $0.69 \mathrm{~V}$ \\
$\mathrm{LSC} 214$ & $0.50 \mathrm{~V}$ & $0.35 \mathrm{~V}$ & $0.85 \mathrm{~V}$ \\
$\mathrm{BSCF}$ & $0.49 \mathrm{~V}$ & $0.26 \mathrm{~V}$ & $0.75 \mathrm{~V}$ \\
$\mathrm{BSCF} \mid \mathrm{LSMO} 8$ & $c a .0 .4 \mathrm{~V}$ & $c a .0 .3 \mathrm{~V}$ & $c a .0 .70 \mathrm{~V}$ \\
\hline
\end{tabular}


Table S5: Calculated $\mathrm{O} p$-band centers of $\mathrm{Sr}_{2} \mathrm{Co}_{1-x} \mathrm{Fe}_{x} \mathrm{O}_{3} \mathrm{Cl}$ relative to the Fermi level

\begin{tabular}{cc}
\hline Composition & $\mathrm{O} p$-band center $[\mathrm{eV}]$ \\
\hline$x=0$ & -2.6 \\
$x=0.25$ & -2.8 \\
$x=1.0$ & -2.7 \\
\hline
\end{tabular}

Table S6: Calculated energies, zero point energy and entropy correction values of OER steps on $\mathrm{Sr}_{2} \mathrm{Co}_{1-x} \mathrm{Fe}_{x} \mathrm{O}_{3} \mathrm{Cl}$ (in eV)

\begin{tabular}{|c|c|c|c|c|}
\hline & $E(x=0.0)$ & $E(x=0.25)$ & $\mathrm{ZPE}^{\mathrm{a}}$ & $T \Delta S \mathrm{~b}$ \\
\hline$*$ & -459.89 & -466.74 & 0 & 0 \\
\hline $\begin{array}{c}\mathrm{OH}^{*} \\
(\mathrm{Co} \text { or } \mathrm{Fe})\end{array}$ & -469.55 & $\begin{array}{l}-476.48 \\
-476.53\end{array}$ & 0.37 & 0 \\
\hline $\begin{array}{c}\mathrm{O}^{*} \\
(\mathrm{Co} \text { or } \mathrm{Fe})\end{array}$ & -464.38 & $\begin{array}{l}-471.31 \\
-471.16\end{array}$ & 0.06 & 0 \\
\hline $\begin{array}{c}\mathrm{OOH}^{*} \\
(\mathrm{Co} \text { or } \mathrm{Fe})\end{array}$ & -473.80 & $\begin{array}{l}-480.72 \\
-471.42\end{array}$ & 0.44 & 0 \\
\hline $\mathrm{H}_{2} \mathrm{O}$ & -14.02 & & 0.57 & 0.67 \\
\hline $\mathrm{H}_{2}$ & -6.80 & & 0.35 & 0.403 \\
\hline
\end{tabular}
a: Reference S9
b: Reference S10 
Table S7: Diffusion coefficients of $\mathrm{Sr}_{2} \mathrm{Co}_{1-x} \mathrm{Fe}_{x} \mathrm{O}_{3} \mathrm{Cl}$

\begin{tabular}{cl}
\hline Composition & $D\left[\mathrm{~cm}^{2} \mathrm{~s}^{-1}\right]$ \\
\hline$x=0$ & $9.6 \times 10^{-12}$ \\
$x=0.1$ & $6.7 \times 10^{-12}$ \\
$x=0.2$ & $6.5 \times 10^{-12}$ \\
$x=0.3$ & $8.6 \times 10^{-13}$ \\
$x=0.5$ & $1.1 \times 10^{-13}$ \\
$x=1.0$ & $8.7 \times 10^{-15}$ \\
\hline
\end{tabular}

Table S8: Electronic resistivities of $\mathrm{Sr}_{2} \mathrm{Co}_{1-x} \mathrm{Fe}_{x} \mathrm{O}_{3} \mathrm{Cl}$

\begin{tabular}{cl}
\hline Composition & $\rho[\Omega \mathrm{cm}]$ \\
\hline$x=0$ & $4 \times 10^{1}$ \\
$x=0.2$ & $2 \times 10^{2}$ \\
$x=0.5$ & $2 \times 10^{3}$ \\
$x=0.8$ & $4 \times 10^{4}$ \\
$x=1.0$ & $4 \times 10^{6}$ \\
\hline
\end{tabular}




\section{References}

(S1) Taguchi, H.; Yamada, S.; Nagao, M.; Ichikawa, Y.; Tabata, K. Surface characterization of $\mathrm{LaCoO}_{3}$ synthesized using citric acid. Mater. Res. Bull. 2002, 37, 69-76.

(S2) Fabbri, E.; Mohamed, R.; Levecque, P.; Conrad, O.; Köts, R.; Schmidr, T. J. $\mathrm{Ba}_{0.5} \mathrm{Sr}_{0.5} \mathrm{Co}_{0.8} \mathrm{Fe}_{0.2} \mathrm{O}_{3-\delta}$ perovskite activity towards the oxygen reduction reaction in alkaline media. ChemElectroChem 2014, 1, 338-342.

(S3) Momma, K.; Izumi, F. VESTA 3 for three-dimensional visualization of crystal, volumetric and morphology data. J. Appl. Cryst. 2011, 44, 1272-1276.

(S4) Ma, X.; Carneiro, J. S. A.; Gu, X.-K.; Qin, H.; Xin, H.; Sun, K.; Nikolla, E. Engineering complex, layered metal oxides: high-performance nickelate oxide nanostructures for oxygen exchange and reduction. ACS Catal., 2015, 5, 4013-4019.

(S5) Van Buren, F. R.; Broers, G. H. J.; Bouman, A. J.; Boesveld, C. An electrochemical method for the determination of oxygen ion diffusion coefficients in $\mathrm{La}_{1-x} \mathrm{Sr}_{x} \mathrm{CoO}_{3-y}$.J. Electroanal. Chem. 1978, 87, 389-394.

(S6) Takeda, Y.; Kanno, R.; Takada, T.; Yamamoto, O.; Takano, M.; Bando, Y. Phase relation and oxygen-non-stoichiometry of perovskite-like compound $\mathrm{SrCoO}_{x}(2.29<x<2.80)$. Z. Anorg. Allg. Chem. 1986, 540, 259-270.

(S7) Mefford, J. T.; Rong, X.; Abakumov, A. M.; Hardin, W. G.; Dai, S.; Kolpak, A. M.; Johnston, K. P.; Stevenson, K. J. Water electrolysis on $\mathrm{La}_{1-x} \mathrm{Sr}_{x} \mathrm{CoO}_{3-\delta}$ perovskite electrocatalysts. Nat. Commun., 2016, 7, 11053.

(S8) Risch, M.; Stoerzinger, K. A.; Maruyama, S.; Hong, W. T.; Takeuchi, I.; Shao-Horn, Y. $\mathrm{La}_{0.8} \mathrm{Sr}_{0.2} \mathrm{MnO}_{3-\delta}$ decorated with $\mathrm{Ba}_{0.5} \mathrm{Sr}_{0.5} \mathrm{Co}_{0.8} \mathrm{Fe}_{0.2} \mathrm{O}_{3-8}$ : a bifunctional surface for oxygen electrocatalysis with enhanced stability and activity. J. Am. Chem. Soc. 2014, 136, 5229-5232.

(S9) Bajdich, M.; García-Mota, M.; Vojvodic, A.; Nørskov, J. K.; Bell, A. T. Theoretical investigation of the activity of cobalt oxides for the electrochemical oxidation of water. J. Am. Chem. Soc. 2013, 135, 13521-13530.

(S10) Haynes, W. M. CRC Handbook of Chemistry and Physics; CRC Press: Boca Raton, FL, 2013. 